\title{
Aplicación de la técnica de componentes principales en la determinación de variables de activos ambientales
}

\author{
Laura Gallego', Ludivia Hernandez', Hanna Orjuela² y Oscar Araque ${ }^{3 *}$ \\ (1) Facultad de Contaduría Pública, Universidad Cooperativa de Colombia, sede Ibagué - Espinal 730001, Colombia \\ (correo-e: laura.gallego@campusucc.edu.co, ludivia.hernandez@campusucc.edu.co) \\ (2) Universidad del Tolima, Ibagué, Ibagué 730001, Colombia (correo-e: hmorjuelaa@ut.edu.co) \\ (3) Departamento de Ingeniería Mecánica, Universidad de Ibagué, Ibagué 730001, Colombia \\ (correo-e: oscar.araque@unibague.edu.co)
}

* Autor a quien debe ser dirigida la correspondencia.

Recibido Jun. 5, 2020; Aceptado Ago. 3, 2020; Versión final Ago. 20, 2020, Publicado Dic. 2020

\begin{abstract}
Resumen
El propósito de este estudio es establecer la relación existente entre los activos ambientales y las variables que influyen en su desarrollo. Se utiliza la técnica de análisis multivariado de clusters o conglomerados. A partir del método Ward para agrupación de clúster e intervalo de distancia euclídea cuadrado, se identifican los grupos que se forman en las dinámicas de los activos ambientales en el marco de la economía del conocimiento y el grado de correlación entre los mismos. Los resultados muestran que existe una fuerte asociación entre los niveles de capacitación y el acceso a la tecnología, y entre las decisiones de gobierno con los sectores que mayor influencia ejercen en los activos ambientales. Se concluye que esto puede entenderse como un indicativo de la fuerte y positiva influencia que tiene la sofisticación tecnológica en la conservación de los recursos ambientales, ligada a las políticas públicas que favorezcan su aplicación y promuevan la inversión en innovación y desarrollo tecnológico.
\end{abstract}

Palabras clave: economía del conocimiento; análisis de cluster; innovación; desarrollo tecnológico

\section{Application of the principal component technique in the determination of environmental asset variables}

\begin{abstract}
The purpose of this research study is to determine the relationships between environmental assets and the variables that influence their development. A multivariate cluster analysis technique is used. The Ward method for clustering and the Euclidean squared distance interval were applied to identify groups formed in environmental asset dynamics in the knowledge economy framework and to determine correlation degrees between them. The results show that there is a strong association between training levels and access to technology and between government decisions and the sectors that have the greatest influence on environmental assets. It is concluded that this is an indication of the strong and positive influence that technological sophistication has on the conservation of environmental resources, which is linked to public policies that favor its application and promote investment in innovation and technological development.
\end{abstract}

Keywords: knowledge economy; cluster analysis; innovation; technological development 


\section{INTRODUCCIÓN}

El desarrollo de la sociedad moderna y la globalización han propiciado el consumo y a la vez el uso de recursos naturales, generando la degradación de los ecosistemas y haciendo cada vez más compleja la sostenibilidad; situación que lleva a las organizaciones necesariamente a reconocer el valor de los activos y recursos ambientales, para de esta manera conseguir fomentar su protección y preservación, así como cuantificar las buenas prácticas en la utilización de los mismos (Anzar y Estruch, 2015). El consumo desmedido de los recursos naturales y la despreocupación por los efectos generados producto del modelo de desarrollo vigente en relación con el medio ambiente, han sido la marca de las prácticas de los sistemas de producción y de los modelos de desarrollo adoptados en los diferentes países del mundo, la atmósfera es esencial para la vida sobre el planeta (Villaflor et al. 2008), sin embargo, el hombre descarga en ella muchos de los residuos producidos por su actividad. Sin embargo, también es cierto que la concientización de las sociedades en lo referente a lo medioambiental, considerándola como elemento esencial de la gestión industrial, consolidando esta dimensión ambiental como elemento de gran interés para el desarrollo de los procesos al interior de las organizaciones (Gomero et al. 2004).

En esta línea, se pueden resaltar los adelantos tecnológicos que se desarrollan desde la ingeniería, avances que buscan mejorar la relación de aprovechamiento de recursos; y los nuevos enfoques que se dan en el ámbito económico, que ya no solo se centra en lo financiero, sino que también busca tener en cuenta aspectos sociales, políticos, culturales, y naturales (Martín et al., 2013). Es por todo lo anterior que la economía ambiental está presentando alternativas, metodologías y soluciones con el fin de medir el impacto que está generando el hombre asociado al crecimiento económico y los adelantos tecnológicos, de manera que consiga mitigar sus efectos adversos (Ucles, 2006).

Al momento de tomar decisiones uno de los factores de mayor incidencia en la dinámica de las organizaciones son las personas (Barrios et al., 2020). La ejecución de estrategias para la protección ambiental ayuda a las pequeñas y medianas empresas, en este sentido, desde la perspectiva ambiental, se plantea que registrar los hechos económicos relacionados con el capital natural entre los cuales se distingue el rubro relacionado con los activos ambientales, resulta muy favorable para garantizar la sostenibilidad en los procesos que adelantan las organizaciones (Martín et al., 2010). No obstante, calcular este deterioro genera algunas dificultades, puesto que está relacionado con la medición de la degradación del entorno, representando por ejemplo en aspectos intangibles como pueden ser el deterioro de la salud y calidad de vida de las personas (Dedeoglu y Dedeoglu, 2020).

Los activos ambientales, son aquellos que no son producidos, por el hombre, pero cumplen la función de proveedores de servicios ambientales y por tanto, apoyan la cadena de producción, como puede ser la absorción de diseños o cualquier otra función del medio ambiente (Zickgraf, 2019). Al respecto (Espinoza et al., 2014), expone que un activo es un derecho de propiedad otorgado a un individuo o un grupo sobre un bien o un recurso; en este sentido, expresa que el titular de un derecho de propiedad puede excluir a terceros que pretendan apropiarse del bien sobre el cual se asienta el derecho. En este orden de ideas, se argumenta que el medio ambiente es un "macro bien", y como tal es un sistema, lo cual significa que es más que sus partes: es la interacción de todas ellas.

La ganancia obtenida por la explotación de activos ambientales es caracterizada por generar renta en actividades que involucren temas ambientales, por ejemplo, la adquisición o implementación de mejoras que se relacionen con esta finalidad (Geba et al., 2010). La importancia de considerar los activos ambientales radica en el reconocimiento que recibe una organización, producto del manejo que le da a su activo medioambiental, desde la perspectiva de involucrar tecnología de punta, el registro de las condiciones y cambios en los recursos naturales y del ambiente, diseño e implementación de indicadores de evaluación de la sostenibilidad ambiental y su desarrollo, todas medidas que tienen como propósito fundamental propiciar la consolidación de sistemas de información que faciliten el control y fiscalización de las acciones que afectan la condición de la naturaleza y el desarrollo (Ziolkowska, 2016).

El estudio de la influencia de los activos ambientales ha sido objeto de investigación ampliamente abordado en años recientes; con relación a lo anterior, los investigadores Ray y asociados (2011), han realizado estudios donde identifican la correspondencia existente entre las inversiones verdes, los precios del mercado y el vínculo entre las reservas de energía limpia y los precios del petróleo crudo, principalmente en empresas que utilizan procesos de producción ecológicos y desarrollan infraestructuras verdes. Los resultados indican el interés creciente de las empresas por migrar hacia activos medioambientales, lo que implica que existen estados de alta y baja volatilidad para los mismos, dado que la creciente preocupación por el cambio climático y la seguridad energética inspiraría a los participantes en el mercado a un cambio hacia inversiones éticas, concluyendo que es algo de gran interés para los inversores que deseen eliminar el carbono de sus carteras incluyendo más activos ambientales. 
Se ha logrado establecer que la huella de carbono es una herramienta importante al momento de evaluar la seguridad ecológica basándose en las necesidades básicas de biomasa de la humanidad (Bi et al. 2020), se logró determinar que la biocapacidad renovable y la huella de carbono han aumentado, mostrándose una marcada diferencia entre las zonas urbanas y rurales, de igual forma el consumo de recursos se concentró en las ciudades con un alto flujo de personas, materiales y turismo, lo cual ha provocado que en ciudades densamente pobladas el nivel de cambie de saludable a no saludable. Lo cual ha provocado que el déficit ecológico renovable y la presión ecológica renovable han aumentado, y los riesgos ecológicos se expanden aún más, por tal motivo es necesario proteger los activos naturales, reducir el consumo ecológico y mejorar la seguridad ecológica y la sostenibilidad.

\section{OTROS ANTECEDENTES}

La sociedad luego de la revolución industrial se vio inmersa en cambios significativos para el desarrollo económico y social, orientados a la mejora de maquinaria y tecnología para la realización de las labores cotidianas, constituyéndose en herramientas para cada día mejorar las condiciones de vida de las personas, dejando de lado que todos estos cambios provocan afectaciones significativas y de distinta índole en el entorno o medio ambiente (Corradini, 2019). Gracias a la implementación de la tecnología, se ha favorecido la eficiencia en las labores realizadas por el hombre cada día pues toman menos tiempo al ser más tecnificadas, y en este sentido, es importante tener presente que se están generando avances considerables y desarrollos tecnológicos a diario, debido a que los adelantos tecnológicos, en especial las tecnologías digitales, se han incorporado y forman parte de las actividades desarrolladas en las empresas y de la vida diaria de las personas (Colding et al., 2019).

Los procesos de innovación tecnológica de acuerdo a lo planteado por, (Eyzaguirre et al., 2005), utilizan el conocimiento como elemento fundamental para generar valor y riqueza por medio de su transformación a información, abarca rubros como la educación, investigación y desarrollo, alta tecnología, informática, telecomunicaciones, robótica, nanotecnología e industria aeroespacial. Desde finales del siglo XX, la inversión en capital intangible ha crecido considerablemente incluso en mayor medida que el capital tangible (Eyzaguirre et al., 2005), encontrándose que la economía del conocimiento es un activo primordial de los bienes de capital y mano de obra por medio de cuatro pilares principales, los cuales son el centro de estrategia para el crecimiento de la organización y lograr obtener un beneficio para toda la sociedad por medio de una educación y capacitación general sobre las tendencias que se están manejando (Sánchez and Ríos, 2011).

Es aquí donde los cambios tecnológicos introducen nuevas configuraciones de las actividades económicas, originando una creciente relevancia en los agrupamientos industriales y de los sistemas de innovación nacional cuya interrelación genera nuevas relaciones entre empresas, universidades y el sector público (Casalet, 2004). Hoy por hoy, la tecnología es aplicada en todos los procesos que realiza el hombre, para ello se han implementado herramientas y máquinas que permitan incrementar el control y lograr una comprensión que facilite cada día los procesos, cabe resaltar que es implementada en las áreas más importantes desarrolladas para mejorar las condiciones de vida del hombre, un ejemplo de estas es la medicina (Ogwueleka, 2011).

No obstante, la economía del conocimiento fomenta la cultura de la cooperación que debe tener la sociedad para poder realizar avances que permitan suplir la necesidad de la misma por medio de procesos ayudados por la tecnología (Curvelo et al., 2018). Por lo tanto, se están tomando precauciones con el fin de no hacer daño al planeta y preservar el medio ambiente, ya que la esencia de esta es mejorar la calidad de vida de todas las personas sin distinción alguna (Suwadi, y Jenal, 2019).

Algunos estudios están orientados a mejorar la productividad del carbono, mecanismo eficaz para reducir las emisiones contaminantes, sin entrar en conflicto con el impulso a la prosperidad económica. Para formular y aplicar adecuadamente las políticas de ahorro de energía y reducción de las emisiones de carbono en diversos sectores, es de gran importancia investigar las características de la evolución y los modos de convergencia de la producción del carbono en todos los sectores manufactureros (Xu et al., 2020), encontraron que la productividad de los sectores manufactureros muestra una tendencia creciente en general, acompañada del crecimiento de las emisiones, y que la eficiencia técnica, en lugar del cambio tecnológico, es la principal contribución al aumento, también se indica que el tamaño de las empresas y la estructura de la industria tienen importantes repercusiones en el impacto medioambiental, mientras que las repercusiones de la intensificación del capital y la estructura del consumo de energía son menores. 


\section{METODOLOGÍA}

Para el desarrollo de esta investigación se utiliza la técnica de análisis multivariable de cluster o conglomerados, con el propósito de establecer la relación presente entre las variables para la preservación de los activos ambientales.

\section{Análisis Multivariado}

El análisis multivariado hace referencia a todas aquellas técnicas estadísticas que simultáneamente examinan distintos objetos de investigación, en la evaluación y recopilación de datos estadísticos se utilizan métodos de análisis multivariantes para aclarar y explicar las relaciones entre las diferentes variables que pueden estar asociadas con estos datos (Hair et al., 1999).

El análisis multivariante siempre se utiliza cuando hay más de tres variables involucradas y el contexto de su contenido no está claro. El objetivo es detectar una estructura, por un lado, y verificar los datos de las estructuras por otro. Anteriormente, en las estadísticas se manejaban métodos de prueba y análisis multivariante para descubrir las relaciones causales. Hoy en día, los análisis multivariantes se suelen llevar a cabo mediante el uso de software, esto con el objetivo de hacer frente a las enormes cantidades de datos y al mismo tiempo, controlar las variables modificadas en aplicaciones prácticas como las pruebas de usabilidad. Sin embargo, las pruebas multivariante también pueden contribuir significativamente a mejorar la facilidad de uso a menor escala (Chopra, 2010).

\section{Segmentación Jerárquica}

La segmentación jerárquica es una técnica que persigue distinguir grupos de elementos homogéneos en una población, utilizando una variable dependiente no métrica o métrica y varias variables independientes métricas que actúan como predictoras. También conocida como técnica de árboles de decisión, ya que puede presentarse como un proceso iterativo descendente de partición de la muestra total en sucesivos grupos en virtud del valor adoptado por la variable dependiente, el cual es función de los valores de las variables explicativas (Blanco et al., 2017).

\section{Análisis de Cluster jerárquicos}

Es una técnica multivariable que busca agrupar elementos tratando de lograr la máxima homogeneidad en cada grupo, esta técnica exploratoria diseña datos para revelarlos en variables y sugiere modos potencialmente útiles de agrupar la información recolectada, sin embargo, divide las observaciones en grupos de acuerdo con la proximidad o lejanía de unas con otras (Perez et al., 2004, Price et al., 2006). Para el proceso de evaluación se utiliza la escala de Likert con 5 niveles de respuesta como se indica en la Tabla 1.

Tabla 1: Escala de valoración de Likert

\begin{tabular}{|l|c|}
\hline Nivel de cumplimiento & Valor \\
\hline Totalmente en desacuerdo & 1 \\
\hline Desacuerdo & 2 \\
\hline Neutral & 3 \\
\hline Aceptable & 4 \\
\hline Totalmente de acuerdo & 5 \\
\hline
\end{tabular}

Se realiza una muestra de 60 empresarios del sector industrial, de diversas áreas de producción, los cuales determinan conforme a la escala de valoración la importancia que las variables indicadas en la Tabla 2 tienen en la conservación de los activos ambientales, afectados por el que hacer industrial (Orjuela et al., 2018).

Tabla 2: Variables de influencia de los activos medio ambientales

\begin{tabular}{|l|c|}
\hline Variable de influencia & Acronimo \\
\hline Población educada y calificada & EQP \\
\hline Sistemas de información eficiente & EIS \\
\hline Infraestructura de la información & II \\
\hline Gobernanza & G \\
\hline Sector económico & ES \\
\hline Desarrollo tecnológico & TD \\
\hline
\end{tabular}




\section{RESULTADOS Y DISCUSIÓN}

Se aplica un modelo de encuesta, a una población de sesenta entrevistados, con los cuales se establece el nivel de relación que existe entre la preservación de los activos ambientales y las variables que los influencian. La información obtenida es mostrada en la Tabla 3.

Tabla 3: Variables de respuesta que influyen en los activos ambientales

\begin{tabular}{|l|c|c|c|c|c|c|}
\hline & \multicolumn{7}{|c|}{ Variables } \\
\hline Activos Medioambientales & EQP & EIS & II & G & ES & TD \\
\hline Costo ambiental (EC) & 4.52 & 4.83 & 4.77 & 4.97 & 4.33 & 4.77 \\
\hline Administración del medioambiente (EM) & 4.6 & 4.77 & 4.53 & 4.85 & 4.0 & 3.73 \\
\hline Activos para la mejora del medio ambiente (AEI) & 3.5 & 4.65 & 4.83 & 2.5 & 2.87 & 4.93 \\
\hline Recursos naturales (NA) & 2.8 & 3.77 & 2.4 & 2 & 3.3 & 4.2 \\
\hline Servicios ambientales (ES) & 4.7 & 4.3 & 2.0 & 2.2 & 2.6 & 4 \\
\hline Capital e inversiones para proteger el medioambiente (AIPE) & 4.63 & 4.5 & 4.7 & 4.9 & 4.53 & 4.72 \\
\hline Activos utilizados para preservar el medioambiente (APE) & 4.4 & 4.53 & 4.64 & 4.7 & 4.63 & 4.82 \\
\hline Activos del ecosistema (EA) & 3.3 & 4.7 & 4.8 & 4.63 & 4.2 & 4.2 \\
\hline Activos tangibles e intangibles (TIA) & 3.73 & 3.83 & 4.25 & 4.21 & 3.7 & 3.84 \\
\hline
\end{tabular}

A partir de la información obtenida se construye un gráfico de contorno donde se indica la valoración de la influencia de las variables promediadas, respecto a la influencia que tienen sobre la conservación de los activos ambientales, el cual se muestra en la Figura 1.

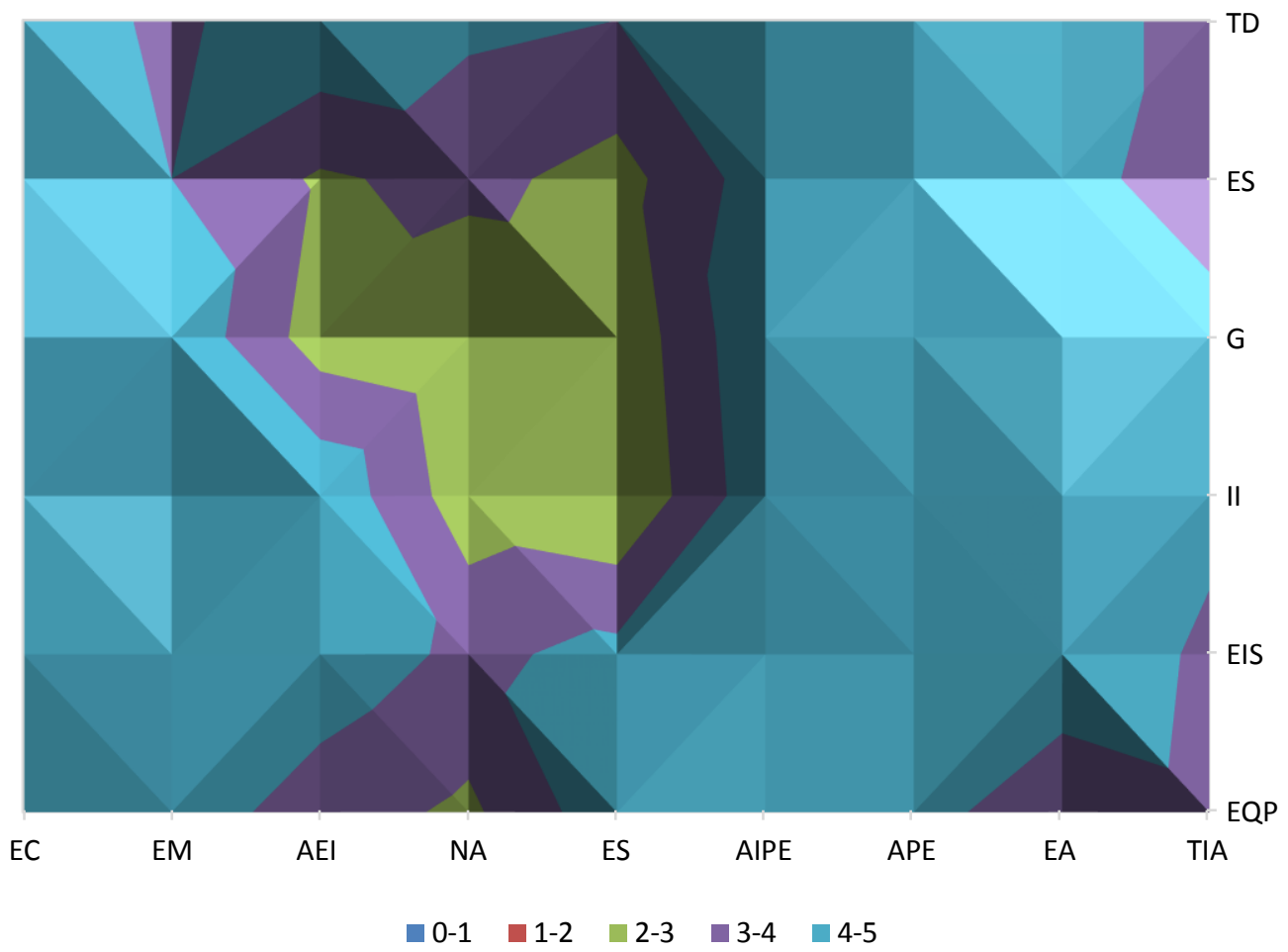

Fig. 1: Contorno de valoración de las variables de influencia.

De lo anterior es posible indicar que existe un alto nivel de relacionamiento entre Sistemas de información eficiente EIS y los activos ambientales, lo cual se debe a la importancia de la información para la conservación de los mismos, de igual forma la relación existente entre gobernanza y capital e inversiones para proteger el medio ambiente (AIPE) se destaca debido a que las políticas estatales o al interior de las organizaciones pueden influenciar en gran medida las acciones frente al accionar de las empresas, el autor Xu et al., (2020) utiliza este enfoque para determinar las principales características que afectan la eficiencia de la administración del carbono 
y la afectación medioambiental, encontrando similitudes con los hallazgos de esta investigación, a la vez que las políticas de gobernanza influyen de forma significativa en el direccionamiento de las organizaciones. Con la información obtenida se realiza un análisis de cluster utilizando el software IBM SPSS, por medio del cual se establece la distancia entre los conglomerados, la medida de la distancia va a ser la distancia euclidea cuya expresión es (1). La matriz de proximidad obtenida con el software es mostrada en la Tabla 4.

$$
d\left(P_{1}, P_{2}\right)=\sqrt{\left(x_{2}-x_{1}\right)^{2}+\left(y_{2}-y_{1}\right)^{2}}
$$

Tabla 4: Matriz de proximidad

\begin{tabular}{|l|r|r|r|r|r|r|}
\hline \multirow{2}{*}{ Evento } & \multicolumn{6}{|c|}{ Variables } \\
\cline { 2 - 7 } & \multicolumn{1}{|c|}{ EQP } & \multicolumn{1}{|c|}{ EIS } & \multicolumn{1}{c|}{ II } & \multicolumn{1}{c|}{ G } & \multicolumn{1}{c|}{ ES } & \multicolumn{1}{c|}{ TD } \\
\hline EQP & .000 & 4.552 & 11.869 & 10.317 & 6.327 & 6.321 \\
\hline EIS & 4.552 & .000 & 7.499 & 12.530 & 7.400 & 1.821 \\
\hline II & 11.869 & 7.499 & .000 & 5.845 & 6.178 & 8.451 \\
\hline G & 10.317 & 12.530 & 5.845 & .000 & 3.706 & 15.648 \\
\hline ES & 6.327 & 7.400 & 6.178 & 3.706 & .000 & 7.372 \\
\hline TD & 6.321 & 1.821 & 8.451 & 15.648 & 7.372 & .000 \\
\hline
\end{tabular}

Conforme a lo anterior es posible obtener, el dendograma enlace ward, distancia euclidea, el cual se muestra en la Figura 2. Aquí se ilustra como las variables que se encuentran más cercanas, se asocian en el mismo cluster, mientras las que menor relación muestran entre si hacen parte de otros cluster

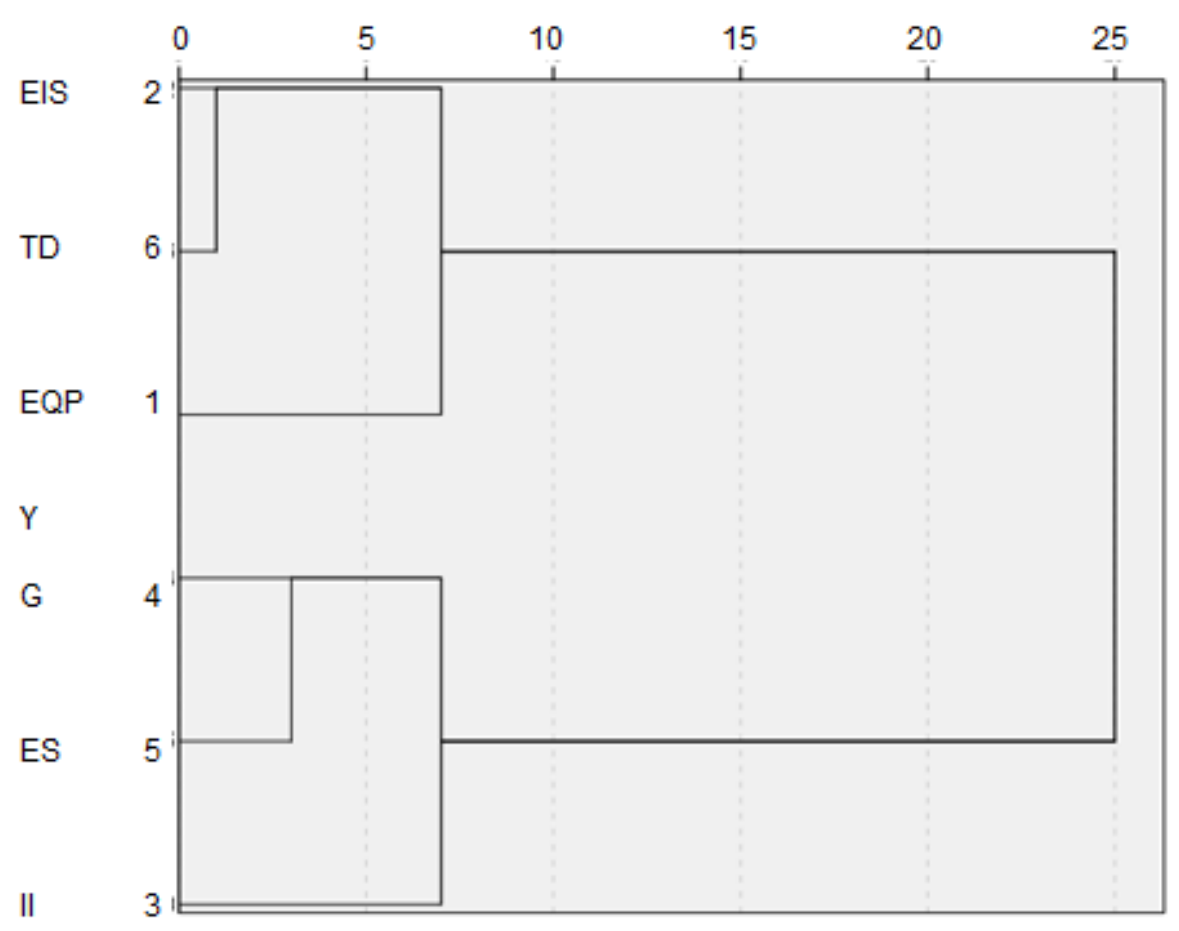

Fig. 2: Dendograma enlace ward, distancia euclidea.

A partir del dendograma expuesto en la Figura 2 y el historial de conglomeración mostrado en la Tabla 5, se observa que los sistemas de información eficientes (EIS) y desarrollo tecnológico (TD) son las primeras aproximaciones que se forman en el historial de cluster, con un nivel de distancia 0.911, seguido por Gobernanza $(\mathrm{G})$ y sector económico (ES) con un nivel de distancia de 2.763, es posible inferir que lo anterior indica que entre estos enlaces se presenta la mayor relación entre variables, por consiguiente, una menor 
distancia, las variables que presentaron un menor nivel de relacionamiento fueron población educada y capacitada (EQP) e infraestructura de la información (II), con una distancia de 19.306.

Además, se observa que se forman dos cluster principales el primero conformado por EIS, TD y EQP, y el segundo formado por G, ES y II, lo cual indica que la población más educada y calificada tiene un mayor acceso a los sistemas de información y desarrollo tecnológico, mientras que los sectores económicos poseen una infraestructura de la información eficiente que le permite a las organizaciones tomar decisiones que inciden en los activos ambientales. Esta técnica ha sido utilizada por el investigador Blanco et al., (2017), demostrando que el análisis jerárquico, facilita determinar el nivel de correlaciona miento entre las diferentes variables, permitiendo generar grupos de asociación, lo cual redunda en una mejor segmentación de los elementos que influencian el aprovechamiento de los activos medioambientales.

Tabla 5: Historial de conglomeración

\begin{tabular}{|c|c|c|c|c|c|c|}
\hline \multirow{2}{*}{ Etapa } & \multicolumn{2}{|c|}{ Cluster combinado } & \multirow{2}{*}{ Coeficiente } & \multicolumn{2}{|c|}{ Primera aparición del grupo de cluster } & \multirow{2}{*}{ Siguiente etapa } \\
\cline { 2 - 3 } & Cluster 1 & Cluster 2 & & Cluster 1 & Cluster 2 & \\
\hline 1 & 2 & 6 & .911 & 0 & 0 & 3 \\
\hline 2 & 4 & 5 & 2.763 & 0 & 0 & 4 \\
\hline 3 & 1 & 2 & 6.084 & 0 & 1 & 5 \\
\hline 4 & 3 & 4 & 9.474 & 0 & 2 & 5 \\
\hline 5 & 1 & 3 & 19.306 & 3 & 4 & 0 \\
\hline
\end{tabular}

Se observa que existe una correlación fuerte entre EIS-TD-EQP y G-ES-II, las variables similares están relacionadas mediante enlaces determinados por el nivel de similitud, de lo anterior se logra entender que la agrupación de variables se da en dos componentes

\section{CONCLUSIONES}

De acuerdo al trabajo presentado y a los resultados obtenidos, se pueden plantear las siguientes conclusiones principales:

1. La técnica de análisis por clusters resulta ser útil para la determinación de las variables de influencia con los activos ambientales, resultando ser de fácil apropiación para los interesados en este método, se logra establecer que las variables que mayor nivel de relacionamiento poseen en relación a la influencia sobre los activos ambientales son Sistemas de información eficientes EIS y Desarrollo tecnológico TD, con un coeficiente de relacionamiento de 0.911 lo cal es un indicativo de la cercanía que poseen estas variables.

2. El relacionamiento entre las variables EIS-TD-EQP indica que existe una fuerte asociación entre los niveles de capacitación y el acceso a la tecnología, lo cual es un indicativo que el acceso a niveles superiores de educación puede llegar a favorecer el relacionamiento con los procesos de sostenimiento y conservación del medioambiente.

3. El relacionamiento entre las variables G-ES-II indica que hay una fuerte asociación entre las decisiones de gobierno tanto al interior de la organización como de políticas y normatividad, y los sectores que mayor influencia pueden llegar a tener en relación a los activos ambientales.

\section{AGRADECIMIENTOS}

Los autores agradecen al departamento de investigaciones de la Universidad Cooperativa de Colombia sede Ibagué - Espinal y de la Universidad de Ibagué, por el apoyo suministrado para el desarrollo de esta investigación, código de proyecto INV 2593.

\section{REFERENCIAS}

Anzar, J., y Estruch Guitart, A. V., Valoracion de activos ambientales teorica y casos. Editorial Universidad Politecnica de Valencia, 2da edicion (2015).

Villaflor, G., Morales, G., y Velasco, J., Variables Significativas del Proceso de Combustión del Gas Natural. Información tecnológica, 19(4), 57-62 (2008).

Gomero, M. D. L., Sáez, P. D. C. Z., Cortés, E. C., y Azorín, J. F. M., La integración del capital medioambiental en el capital intelectual de la empresa. Revista de economía y empresa, 21(50), 11-28 (2004). 
Martín-de Castro, G., Delgado-Verde, M., Navas-López, J. E., y Cruz-González, J., The moderating role of innovation culture in the relationship between knowledge assets and product innovation. Technological Forecasting and Social Change, 80(2), 351-363 (2013).

Ucles Aguilera, D., El valor economico del medio ambiente. Ecosistemas, 15(2), 1-6 (2006).

Barrios, K., Olivero, E., y Figueroa, B., Condiciones de la gestión del talento humano que favorecen el desarrollo de capacidades dinámicas. Información tecnológica, 31(2), 55-62 (2020).

Martín-Tapia, I., Aragón-Correa, J. A., y Rueda-Manzanares, A., Environmental strategy and exports in medium, small and micro-enterprises. Journal of World Business, 45(3), 266-275 (2010).

Dedeoglu, C., y Dedeoglu, C. E., Information Infrastructures and the Future of Ecological Citizenship in the Anthropocene. Social Sciences, 9(1), 3 (2020).

Zickgraf, C., Keeping People in Place: Political Factors of (Im) mobility and Climate Change. Social Sciences, 8(8), 228 (2019).

Espinoza Lozada, J., Matheus López, C., y otros seis autores, Arbitraje de consumo. Revista chilena de derecho, 41(3), 791-823 (2014).

Geba, N. B., Fernández, L. E., y Bifaretti, M. C., Marco conceptual para la especialidad contable socioambiental. Actualidad Contable Faces, 13(20), 49-60 (2010).

Ziolkowska, J. R., Socio-economic implications of drought in the agricultural sector and the state economy. Economies, 4(3), 19 (2016).

Ray, R., Ganguly, D., y otros cinco autores, Carbon sequestration and annual increase of carbon stock in a mangrove forest. Atmospheric Environment, 45(28), 5016-5024 (2011).

Bi, M., Xie, G., y Yao, C. Ecological security assessment based on the renewable ecological footprint in the GuangdongHong Kong-Macao Greater Bay Area, China. Ecological Indicators, 116, 106432 (2020).

Corradini, R. A, Set of State-Space Models at a High Disaggregation Level to Forecast Italian Industrial Production. JMultidisciplinary Scientific Journal, 2(4), 508-560 (2019).

Colding, J., Barthel, S., y Sörqvist, P., Wicked Problems of Smart Cities. Smart Cities, 2(4), 512-521 (2019).

Eyzaguirre, N., Marcel, M., Rodríguez, J., y Tokman, M., Hacia la economía del conocimiento: el camino para crecer con equidad en el largo plazo. Estudios Públicos, 97, 5-57 (2005).

Sánchez, C., y Ríos, H., La economía del conocimiento como base del crecimiento económico en México. EnI@ ce: Revista Venezolana de Información, Tecnología y Conocimiento, 8(2), 43-60 (2011).

Casalet, M., Los desafíos de la economía del conocimiento en el contexto institucional: de la jerarquía a la flexibilidad organizativa. Revista mexicana de sociología, 66(3), 565-588 (2004).

Ogwueleka, F. N., Data mining application in credit card fraud detection system. Journal of Engineering Science and Technology, 6(3), 311-322 (2011).

Curvelo, J. O., García, M., y otros ocho autores, Visiones de la contabilidad en el siglo XXI. «etica aplicada y practicas de sostenibilidad en las empresas, lecturas al contexto colombiano", V. 5, 97-120, Ediciones Nueva Jurídica, Bogota, Colombia (2018).

Suwadi, N. A., y Jenal, R., Analysis of process chain network for book borrowing process. Journal of Engineering Science and Technology, 14(4), 1940-1947 (2019).

$\mathrm{Xu}, \mathrm{R}$. , Wu, Y., y Huang, Y., Measurement and convergence of carbon productivity across Shanghai's manufacturing sectors. International Journal of Climate Change Strategies and Management (2020).

Hair, J. F., Anderson, R. E., Tatham, R. L., y Black, W. C., Análisis multivariante (Vol. 491). Madrid: Prentice Hall. (1999). Chopra, P., Multivariate Testing In Action: Five Simple Steps To Increase Conversion Rates. Smashing Magazine, 24 (2010).

Blanco-Blanco, Á., Muñoz, I. A., Molina, E. C., de Miguel, C. R., y Casas, E. E., Aplicaciones de la segmentación jerárquica en medición y evaluación de programas educativos. Ejemplos con un programa de educación financiera. Educación XX1, 20(2), 235-257 (2017).

Pérez López, S., Manuel Montes Peón, J., y Vázquez Ordás, J. C., Managing knowledge: the link between culture and organizational learning. Journal of knowledge management, 8(6), 93-104 (2004).

Price, A. L., Patterson, N. J., Plenge, R. M., Weinblatt, M. E., Shadick, N. A., y Reich, D., Principal components analysis corrects for stratification in genome-wide association studies. Nature genetics, 38(8), 904 (2006).

Orjuela, H. M., Gallego, L. C., y Hernandez, L., Aproximaciones conceptuales de los activos ambientales en el marco de la economía del conocimiento. ARJÉ. Revista de Postgrado FaCE-UC. 12(22), Enero-Julio 2018, $358-369$ (2018).

Gallego, L., y Araque, O., Variables de Influencia en la Capacidad de Aprendizaje. Un Análisis por Conglomerados y Componentes Principales. Información tecnológica, 30(2), 257-264 (2019). 